\title{
La pronunciación del chino para hispanohablantes. Análisis contrastivo de los rasgos prosódicos distintivos entre chino y español
}

DOI: $10.32870 /$ mycp.v2i4.405

Cao Yufei ${ }^{1}$

\section{Resumen}

Este trabajo tiene como finalidad presentar a hispanohablantes la pronunciación del chino desde el punto de vista de la función social del lenguaje. Basándonos en el análisis contrastivo de los rasgos prosódicos distintivos entre chino y español, proponemos algunos consejos prácticos para mejorar la enseñanza de la fonética del chino a alumnos hispanohablantes.

Palabras clave: rasgo prosódico distintivo, análisis contrastivo entre chino y español, enseñanza de chino a hispanohablantes.

\section{Abstract}

This paper aims to present the Chinese's pronunciation from the perspective of the social function of language. Based on the contrastive analysis of distinctive prosodic features between Chinese and Spanish, we put forward some teaching suggestions to improve the teaching of Chinese's phonics to Spanish-speaking students.

Artículo recibido el 29 de enero de 2013 y dictaminado el 08 de marzo de 2013.

1. Universidad de Estudios Internacionales de Shangai. Correo electrónico: yufeielisa@yahoo.cn 
Keywords: distinctive prosodic feature, contrastive analysis between $\mathrm{Chi}$ nese and Spanish, teaching Chinese to Spanish-speaking people.

\section{Introducción}

El aprendizaje de la pronunciación de una lengua extranjera es básico para comunicarse de manera adecuada y fluida porque si la pronunciación es defectuosa, provocará malentendidos. Este artículo presentará a hispanohablantes la pronunciación del chino ${ }^{2}$ desde el punto de vista de la función social del lenguaje, la de comunicación, y que, por consiguiente, nos llevará a abordar aquí sólo el estudio de los rasgos distintivos. Además, este trabajo se limita al análisis del aspecto prosódico, ya que las investigaciones sobre la pronunciación de la lengua extranjera destacan el papel de los factores

Desde el punto de vista de la fonética auditiva, en la percepción de un estímulo acústico existen dos aspectos de naturaleza muy diferente: uno es el físico, que se puede medir objetivamente en todos sus componentes; otro es el psicológico, es decir, el grado de sensación que ese estímulo produce en nosotros; este aspecto sensorial es subjetivo y mucho más difícil de controlar prosódicos en la percepción del acento extranjero, considerando que éstos contribuyen más a la inteligibilidad de los hablantes no nativos que los aspectos relacionados con la articulación de los sonidos (Jilka, 2000; Munro y Derwing, 1999).

\section{Rasgos distintivos prosódicos y percepción de un estímulo acústico}

En el marco de la fonética acústica, el rasgo distintivo se refiere a las relaciones entre las unidades fónicas distintivas de las lenguas, que se basan en la presencia o ausencia de un rasgo distintivo. Los rasgos distintivos se dividen en dos clases: los rasgos prosódicos y los rasgos intrínsecos o inherentes (Quilis, 1999: 111). Sólo los fonemas que

2. Chino mandarín. 
constituyen el núcleo silábico presentan los rasgos prosódicos y únicamente se pueden definir en función del relieve de la sílaba o de la cadena silábica. Se distinguen tres tipos de rasgos prosódicos: la frecuencia fundamental, la intensidad y la cantidad (que también se denominan, respectivamente, tono, fuerza y cantidad).

Por otro lado, desde el punto de vista de la fonética auditiva, en la percepción de un estímulo acústico existen dos aspectos de naturaleza muy diferente: uno es el físico, que se puede medir objetivamente en todos sus componentes; otro es el psicológico, es decir, el grado de sensación que ese estímulo produce en nosotros; este aspecto sensorial es subjetivo y mucho más difícil de controlar (Quilis, 1999: 137). Además, en los hechos del lenguaje hay que tener en cuenta que la sensación del estímulo físico se canaliza a través de las estructuras de cada lengua. Veamos el siguiente cuadro:

\section{Cuadro 1}

\begin{tabular}{ll}
\hline Dimensión física & Dimensión psicológica \\
\hline Cantidad & Duración de la persistencia del sonido \\
Intensidad & Sonía o intensidad subjetiva \\
Frecuencia fundamental & Tonía, tono o percepción de la altura tonal del sonido \\
Estructura acústica & Timbre o cualidad del sonido \\
\hline
\end{tabular}

Fuente: Quilis, 1999: 138.

Un estímulo acústico cualquiera contiene cuatro elementos físicos constitutivos: cantidad, intensidad, frecuencia fundamental y estructura formántica de las ondas sonoras. Estos elementos físicos se complementan en un patrón complejo de dimensiones psicológicas. La percepción de los sonidos del lenguaje es un complejo de estas dimensiones y el juicio lingüístico depende de su interacción.

Por medio del análisis arriba expuesto descubrimos que los tres tipos de rasgos prosódicos distintivos (frecuencia fundamental, intensidad, cantidad) también se encuentran en la dimensión física de la percepción de un estímulo acústico, y por lo tanto, son elementos fonológicos de mayor importancia tanto acústicamente como en el aspecto auditivo. En el apartado que sigue haremos un estudio contrastivo de estos tres rasgos distintivos. 


\section{Análisis contrastivo de los rasgos prosódicos distintivos entre chino y español}

Metodológicamente, este trabajo se encuadra en la teoría de lingüística contrastiva, que se desarrolló en gran medida a mediados del siglo xx y en un sentido moderno debe su consolidación a Robert Lado: Linguistics Across Cultures (1957). Lado postula que la comparación de la lengua materna (L1) del alumno y la lengua meta (L2) determinará las diferencias y similitudes entre ambas, con lo que se podrán predecir las zonas de dificultad en todos los niveles del sistema de la lengua, lo cual se interpreta, en nuestro caso, como la pronunciación. Por lo tanto, a continuación haremos un estudio contrastivo de los rasgos prosódicos distintivos entre chino y español para encontrar las diferencias así como las causas de la dificultad de la pronunciación del chino para los estudiantes hispanohablantes.

Los estudios contrastivos se dividen en los teóricos y los aplicados, y en torno a la distinción entre los primeros y los últimos, podemos apuntar la opinión de Santos Gargallo (1993), quien afirma:

La diferencia fundamental entre una y otra radica en el objetivo final de la investigación: la versión teórica busca consecuencias en el ámbito de los universales lingüísticos y generalmente compara más de dos lenguas; la versión práctica, sin embargo, busca tanto las diferencias como las similitudes entre pares de lenguas con el propósito de aplicar sus resultados al proceso de aprendizaje-enseñanza de segundas lenguas.

De acuerdo con lo que apunta esta investigadora, nuestro trabajo será un estudio contrastivo aplicado. En primer lugar, comparemos los primeros dos rasgos prosódicos distintivos (frecuencia fundamental o tono, intensidad o fuerza) de las dos lenguas tratadas. La china es una lengua tonal; mientras que en español es el acento (intensidad de la voz) el que desempeña la función de la diferenciación del significado.

Veamos primero los cuatro tonos en chino. El tono es la variación de la altura musical, especialmente el ascenso y el descenso. En el chino mandarín hay cuatro tonos: primer tono "-"3 (alto y sostenido), segundo tono "/"

3. Signo tónico. 
(ascendente de medio a alto), tercer tono " $v$ " (descendente y ascendente) y cuarto tono " $\backslash$ ” (descendente de alto a bajo). Veamos el cuadro 2 :

Cuadro 2

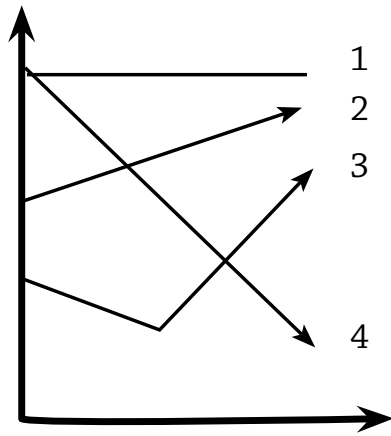

Fuente: Li, 2003.

La diferencia en el tono determina el significado. Por ejemplo, 妈mā: madre; 麻má: cáñamo; 马mă: caballo; 骂 mà: regañar. En el ejemplo citado, la única diferencia de las cuatro palabras monosilábicas estriba en el tono. Hay que resaltar que la altura musical del tono chino es una altura relativa, que no se refiere a la altura absoluta del volumen de la voz de una persona. (Por ejemplo, "Háblame en un tono más alto, porque soy un poco sordo"). La altura musical relativa consiste en la forma de la variación de la altura musical y la amplitud del ascenso y descenso.

El español no es una lengua tonal. En esta lengua, el que desempeña la función de la diferenciación del significado en el nivel prosódico es el acento, que permite poner de relieve una unidad lingüística superior al fonema (sílaba, morfema, palabra, sintagma, frase) para diferenciarla de otras unidades lingüísticas del mismo nivel. Existen dos tipos de acento: acento fijo y acento libre. Por ejemplo, en turco, que es una lengua de acento fijo, el acento recae siempre sobre la última sílaba. El español es una lengua de acento libre. Eso quiere decir que el acento puede ocupar distintas posiciones.

El acento desempeña en la lengua española tres funciones: a) la función contrastiva entre sílabas acentuadas e inacentuadas; b) la función distintiva se realiza en el eje paradigmático en las lenguas de acento libre. Su cambio de situación sirve para distinguir dos unidades de significado diferente. Por ejemplo, paso / pasó; término / termino / terminó; y c) la función culminativa, 
en las lenguas de acento libre señala la presencia de una unidad acentual, sin indicar exactamente los límites.

En cuanto al tercer rasgo prosódico distintivo, hay que apuntar que tanto en chino como en español, la cantidad o duración sólo desempeña la función de la diferenciación del significado en muy pocas ocasiones. Por ejemplo, le / lee. Sin embargo, una gran diferencia entre las dos lenguas en este aspecto consiste en que la duración vocálica inherente en español es más breve. Según Marín (1995), los valores medios de la duración de las vocales en español son entre $60.66 \mathrm{~ms}$ y $69.63 \mathrm{~ms}$; mientras que en chino los mismos valores son entre $139.25 \mathrm{~ms}$ y $181.75 \mathrm{~ms}$ (Xiang, 2012). Por ejemplo, el valor medio de la duración inherente de [a] en chino es de 172.75 ms (Xiang, 2012); mientras que la de [a] en español es de 69.63 ms (Marín, 1995). Por lo tanto, la articulación de "mā mā" (madre en chino) es mucho más larga que "mamá".

\section{Enseñanza fonética del chino a estudiantes hispanohablantes}

Los cuatro tonos en chino hacen que esta lengua suene un poco cantarina, pero agradable para el oído. Como en español no hay tono, en muchas ocasiones los alumnos hispanohablantes no pueden pronunciar los tonos correctamente ni distinguirlos. Por ejemplo, confunden mucho los siguientes pares de palabras: camarada: 同志 tóng zhì / dominar: 统治tǒng zhì; esfuerzo: 努 力nǔ lì / esclavo: 奴隶nú lì; honesto: 正直zhèng zhí / política: 政治zhèng zhì; comprar: 买măi / vender: 卖mài. Otro problema que suelen tener los hispanohablantes es pronunciar el chino demasiado rápido, ya que se han acostumbrado al sistema fonético de español, que cuenta con una duración vocálica relativamente breve. Para mejorar la enseñanza fonética del chino a hispanohablantes, proponemos los siguientes consejos prácticos.

Primero, al principio del aprendizaje se recomienda pronunciar los tonos de manera exagerada, destacando sus diferencias. Una vez lograda la distinción clara de los tonos les podríamos exigir más la naturalidad de la pronunciación o realización fonética.

En segundo lugar, no tienen que confundir el tono en chino con el acento en español. El primero es la variación de la altura musical, mientras que el segundo implica una pronunciación más fuerte o de mayor intensidad de una sílaba en una palabra. 
En tercer lugar, hay que prestar atención a los siguientes detalles:

1. Signo tónico: el signo tónico se coloca sobre la principal (la más fuerte) de la sílaba. Por ejemplo, en $o$ de 同tong cae el segundo tono: tóng.

2. Tono ligero: ${ }^{4}$ algunas sílabas, cuando se ponen después de las otras, pierden su propio tono y se pronuncian breves y débiles, dando lugar a lo que se denomina tono ligero. El tono ligero no lleva ningún signo tónico.

3. Cambio de tono: los tonos de varias sílabas pronunciadas en sucesión son, a veces, diferentes de los de las sílabas pronunciadas por separado. Por ejemplo, cuando un tercer tono precede a otro igual, el primero de ellos se convierte en tono segundo: nĭ + hăo ${ }^{\circledR}$ ní hăo. ${ }^{5}$

Cuando un tercer tono precede a un tono primero, segundo, cuarto y a la mayoría de los tonos ligeros, se convierte en un semitercer tono, es decir sólo se pronuncia su primera mitad del tono original (tono descendente): lăo $+\operatorname{sh}^{\circledR}$ lao (semitercer tono) shi. ${ }^{6}$

Cuando "bù" (no) precede al cuarto tono, se convierte en el segundo tono: bù + shì ${ }^{\circledR}$ bú shì. ${ }^{7}$ Se debe poner el signo tónico original de la sílaba en la escritura cuando hay cambios de tono en la pronunciación.

Por último, hay que tener en cuenta que la duración vocálica en chino es más larga. Por lo tanto, al aprender a hablar chino, los estudiantes procedentes de los países hispanohablantes deben alargar la pronunciación de las vocales, tratando de articular con mayor claridad cada sílaba.

\section{Conclusión}

A los alumnos hispanohablantes, al ser nativos de una lengua no tonal, les representa un gran esfuerzo adquirir las destrezas prosódicas de una lengua tonal, como es el caso del chino mandarín. Por lo tanto, en la enseñanza de la pronunciación del chino a hispanohablantes han de primar los contenidos prosódicos. Cuando aprenden a hablar chino, estos alumnos deben ser conscientes de las diferencias entre el chino y el español en cuanto a los rasgos prosódicos distintivos. En la primera etapa del aprendizaje de chino es po-

\footnotetext{
4. Término en chino: 轻声.

5. 你好: hola.

6. 老师: maestro.

7. 不是: no ser.
} 
sible pronunciar los tonos y alargar la articulación de las vocales de manera exagerada. Una vez que logren distinguir los tonos y tengan la conciencia de que la duración vocálica en chino es más larga, podremos exigir más la fluidez y la naturalidad.

\section{Referencias bibliográficas}

Jilka, M. (2000), The contribution of intonation to the perception of foreign accent, Stuttgart: Stuttgart University.

Lado, Robert (1957), Linguistics across cultures: Applied linguistics for language teachers, Ann Arbor: University of Michigan Press.

Li, Weiji (2003), Chino para hispanohablantes, México DF: Universidad Nacional Autónoma de México.

Lu, Jingsheng (1991), “Comparación entre la fonética china y española”, Lenguas Extranjeras, núm. 76, pp. 58-61.

Marín, Rafael (1995), “La duración vocálica en español”, ELUA, núm. 10, pp. 213-226.

Munro, M. J., y Derwing, T. M. (1999), "Foreign accent, comprehensibility, and intelligibility in the speech of second language learners", Language Learning, 49(1), pp. 285-310.

Quilis, Antonio (1999), Tratado de fonología y fonética españolas, Madrid: Gredos.

Santos Gargallo, Isabel (1993), Análisis contrastivo, análisis de errores e interlengua en el marco de la lingüística contrastiva, Madrid: Síntesis.

Xiang, Ning (2012), "Estudio experimental sobre la duración vocálica en el chino mandarín”, Revista Académica de Jinan, núm. 164, pp. 124-129. 